\title{
Long-term Safety of Asenapine in Pediatric Patients Diagnosed With Bipolar I Disorder: A 50-Week Open-Label, Flexible-Dose Trial
}

\author{
Robert L. Findling ${ }^{1} \cdot$ Ronald L. Landbloom ${ }^{2} \cdot$ Mary Mackle $^{2} \cdot$ Xiao $\mathrm{Wu}^{3}$ • \\ Linda Snow-Adami ${ }^{2} \cdot$ Kiki Chang $^{4} \cdot$ Suresh Durgam $^{3}$
}

Published online: 26 July 2016

(c) The Author(s) 2016. This article is published with open access at Springerlink.com

\begin{abstract}
Background Sublingually administered asenapine was approved in March 2015 by the United States Food and Drug Administration for patients aged 10-17 years with an acute manic or mixed episode associated with bipolar I disorder (BP-1). This is the first long-term safety and tolerability study of asenapine in this population.

Methods Following the 3-week randomized, double-blind, placebo-controlled trial of patients aged 10-17 years with an acute manic or mixed episode associated with BP-1, patients could enroll in this flexible-dose (2.5-10 mg twice daily) open-label extension (OLE) study for an additional 50 weeks, conducted from August 2011 to September 2014 in the United States and Russia. Treatment-emergent adverse events (TEAEs) were assessed and predefined
\end{abstract}

Electronic supplementary material The online version of this article (doi:10.1007/s40272-016-0184-2) contains supplementary material, which is available to authorized users.

Robert L. Findling

RFindli1@jhmi.edu

1 Johns Hopkins University and The Kennedy Krieger Institute, Baltimore, MD, USA

2 Merck, Whitehouse Station, NJ, USA

3 Allergan, Jersey City, NJ, USA

4 Lucille Packard Children's Hospital, Stanford University School of Medicine, Stanford, CA, USA
TEAEs of interest reported in addition to metabolic and anthropometric parameters. The Young Mania Rating Scale (YMRS) and Clinical Global Impressions scale in bipolar illness (CGI-BP) were used to assess effectiveness. Results A total of 321 patients (lead-in study treatment: placebo, $n=80$; asenapine, $n=241$ ) were included; 267 $(83.2 \%)$ reported one or more TEAE and $181(56.4 \%)$ discontinued early, $48(15.0 \%)$ due to TEAEs. Of the predefined TEAEs of interest, combined somnolence/sedation/hypersomnia occurred most frequently (42.4\%) followed by oral hypoesthesia/dysgeusia $(7.5 \%)$. In total, $109(34.8 \%)$ patients experienced clinically significant weight gain ( $\geq 7 \%$ increase). No clinically meaningful changes were noted for laboratory parameters measured. Eighteen patients met the criteria for new-onset metabolic syndrome (MBS) post-baseline during the extension study, whereas 10 patients who met MBS criteria at baseline did not meet MBS criteria at endpoint. A total of 12 patients met MBS at baseline and endpoint. Mean change in YMRS total score from OLE baseline was -9.2 points at week 50, and change in CGI-BP severity overall score was similar among all treatment groups (those who initially received asenapine and those who initially received placebo). After 26 weeks of treatment in the OLE, $79.2 \%$ of patients were classified as YMRS $50 \%$ responders relative to acute trial baseline.

Conclusions Asenapine was generally well tolerated in pediatric patients with BP-1 during $\leq 50$ weeks of openlabel treatment; among predefined TEAEs of interest, the combination of somnolence/sedation/hypersomnia was the most common.

Trial registration ClinicalTrials.gov: NCT01349907. 


\section{Key Points}

Long-term flexible dose administration of sublingual asenapine was generally well tolerated in pediatric patients with a manic or mixed episode associated with bipolar I disorder.

Fifteen percent of patients discontinued due to treatment-emergent adverse events; clinically significant weight gain was experienced by $34.8 \%$ of patients.

Among the predefined treatment-emergent adverse events of interest, the combination of somnolence, sedation, and hypersomnia was most frequent (42.4\% of patients).

\section{Introduction}

Many patients with bipolar I disorder (BP-1) experience onset of mania prior to adulthood, and evidence suggests that BP-1 is relatively common in pediatric-aged patients; a cross-sectional survey of more than 10,000 American adolescents aged 13-18 years identified in household and school settings estimated that the lifetime prevalence of BP-1 was $1.7 \%$ [1]. These data are similar to results of an earlier meta-analysis of more than 16,000 young people aged 7-21 years, which estimated the lifetime prevalence of BP-1 to be $1.8 \%$, with no difference observed between US and non-US populations [2]. Freeman and colleagues [3] reported that the quality of life associated with pediatric bipolar disorder is more burdensome than a variety of physical conditions and appears to have a greater impact than other pediatric psychiatric conditions.

Individuals who experience BP-1 earlier in life are expected to develop a more chronic, severe, and recurrent course of the disease when compared with individuals who developed the disorder in adulthood [4-7]. Bipolar disorder is a major cause of disability in young people [8].

Asenapine is a sublingually administered second-generation atypical antipsychotic. In the United States, asenapine was approved in adults for use as monotherapy (August 2009) or adjunctive therapy with lithium or valproate (September 2010) for manic or mixed episodes associated with BP-1. In March 2015, asenapine was approved by the US Food and Drug Administration (FDA) for use as monotherapy in pediatric patients aged 10-17 years with an acute manic or mixed episode associated with BP-1 [9]. Asenapine is not approved for the treatment of pediatric patients with BP-1 outside of the US, but is approved or registered in 65 countries as monotherapy for the treatment of adults with BP-1.
Because BP-1 is a chronic illness and a serious condition, long-term treatment is usually necessary. There are presently no long-term data available on the safety and tolerability of asenapine in pediatric patients with BP-1 of which we are aware. In general, there are limited empirical data that describe the long-term safety of any secondgeneration antipsychotics in pediatric patients. Completed studies have shown evidence that sedation, hyperprolactinemia, and metabolic abnormalities may develop during pharmacotherapy with this class of medications [10]. Therefore, in this study, we sought to characterize the safety of asenapine over 50 weeks in an open-label extension (OLE) trial.

\section{Methods}

Details of the antecedent 3-week, randomized, doubleblind, placebo-controlled efficacy and safety trial of asenapine in pediatric patients with an acute manic or mixed episode associated with BP-1 (NCT01244815) have been previously published [11]. In brief, patients were randomized 1:1:1:1 to placebo, asenapine $2.5,5$, or $10 \mathrm{mg}$ twice daily (bid) for 3 weeks (see electronic supplementary material [ESM], Online Resource 1). After completing the acute trial, patients could enroll in this 50-week OLE trial. The first day of the OLE trial overlapped with day 21/endof-treatment period for the acute mania trial. The OLE was a flexible-dose, multicenter trial to evaluate the long-term safety and tolerability of asenapine monotherapy in pediatric patients aged 10-17 years with a manic or mixed episode associated with BP-1 (NCT01349907); the trial was conducted from August 16, 2011 to September 5, 2014, in the United States and Russia. Diagnosis of BP-1 was made according to the Diagnostic and Statistical Manual of Mental Disorders, fourth edition, text revision (DSM-IV-TR) with current manic (296.4x) or mixed (296.6x) episode with or without psychotic features required and confirmed by a structured clinical interview which consisted of a medical and psychiatric history, DSM-IV-TR checklist, and the Kiddie-Schedule for Affective Disorders and Schizophrenia-Present and Lifetime Version at screening before enrollment in the acute trial.

Independent Ethics Committees associated with each study site reviewed and approved the protocol and applicable amendments. The study was conducted in accordance with Good Clinical Practice standards and applicable country and/or local statutes and regulations regarding ethical committee review, informed consent, and the protection of human patients participating in biomedical research. Written informed consent was provided by the patient's parent, legal representative, or caretaker and 
written patient assent was also obtained before any trial procedures or assessments were performed. If at any time during the patient's participation they turned 18 years of age, they were asked to provide written informed consent. This was an open-label trial and therefore did not require the use of blinding procedures although the blind was maintained with respect to the acute phase trial until that database was unlocked. An external Data Monitoring Committee met regularly to evaluate safety data in an unblinded fashion during the course of the trial. The OLE was initially designed as a 26-week study and amended to 50 weeks to meet European Medicines Agency requirements. The protocol was also amended to include subjects of younger age (10 and 11 years) and older age (18 years of age). Although patients aged 18 years were permitted to enroll in the OLE, predefined analyses were limited to ages $\leq 17$ at OLE entry; the one patient who was aged 18 at entry was therefore excluded from analyses.

\subsection{Inclusion Criteria}

Patients aged 10-18 years who both completed the acute trial and who, in the investigator's opinion, could potentially benefit from long-term asenapine treatment were eligible for enrollment. Key inclusion criteria from the acute trial included a primary diagnosis of BP-1 according to the DSM-IV-TR with a current manic $(296.4 \mathrm{x})$ or mixed (296.6x) episode with or without psychotic features (confirmed by a structured clinical interview that consisted of a medical and psychiatric history, DSM-IV-TR checklist, and the Kiddie-Schedule for Schizophrenia and Affective Disorders-Present and Lifetime Version at screening), a Young Mania Rating Scale (YMRS) score $\geq 20$, and an overall severity of bipolar mania and depression $\geq 4$ on the Clinical Global Impressions scale in bipolar illness (CGIBP). For enrollment in the OLE, patients were required to demonstrate an acceptable level of adherence to antecedent trial medication, study visits, and other protocol requirements in the acute trial according to the investigators' opinion, and to have a caregiver living with them able to provide continued support for adherence to the study protocol.

\subsection{Exclusion Criteria}

Patients with a pervasive development disorder, schizophrenia, schizoaffective disorder, post-traumatic stress disorder, obsessive-compulsive disorder, or psychosis due to a medical condition or prohibited concomitant medication were excluded from the acute trial. Patients with a positive urine pregnancy test at OLE baseline or with the intention to become pregnant during the trial were excluded. Additional criteria that warranted exclusion from the OLE included being at imminent risk of self-harm or harm to others, in the investigator's opinion, based on clinical interview and responses provided on the Columbia Suicide Severity Rating Scale (C-SSRS), involuntary inpatient commitment, poor adherence to the acute trial protocol, and known serological evidence of human immunodeficiency virus antibody. In addition, patients with an uncontrolled, unstable, clinically significant medical condition were excluded. Prohibited concomitant medications included all psychotropic drugs with the exception of stimulants (for attention-deficit hyperactivity disorder [ADHD]) and short-acting benzodiazepines (for transient agitation, irritability, restlessness, insomnia, and hostility). Anticholinergics were also allowed as a rescue medication for extrapyramidal symptoms (EPS).

\subsection{Dosing}

All patients, whether treated with asenapine or placebo in the acute trial, were administered open-label asenapine formulated as a fast-dissolving, black-cherry-flavored sublingual tablet at $2.5 \mathrm{mg}$ bid on day 1 . On the evening of day 4 , the asenapine dose was increased to $5 \mathrm{mg}$ bid. At the day 7 visit, the asenapine dose was increased to $10 \mathrm{mg}$ bid, if tolerated, beginning with the evening dose. The dose was increased sooner, at the discretion of the investigator, if clinically warranted. From day 7 onward, asenapine dosing was flexible based on tolerability and/or symptomatology. Study visits also occurred on days 14, 28, 42, 70, 98, 126, 154, 182, 210, 238, 266, 294, 322, and 350 (see ESM, Online Resource 1).

\subsection{Safety Outcomes}

There were no prespecified key safety endpoints; exploratory safety events and tolerability parameters were assessed throughout the trial (i.e., vital signs were recorded at every study visit; laboratory tests were administered at visits 14 , $18,20,25$, and 28 [i.e., days 28, 126, 182, 266, 350]; and anthropometric parameters were assessed at visits 13,14 , 16,18 , and 20 through 28 [i.e., days 14, 28, 70, 126, and 182-350]). Adverse events were recorded based on spontaneous report or investigator observation; reported adverse events were monitored with the relevant clinical assessments and/or laboratory tests. An adverse event was considered treatment emergent (TEAE) if it was newly reported after open-label baseline or reported to have worsened in severity since open-label baseline. Predefined TEAEs of interest in the study were akathisia, dizziness, insomnia, somnolence/hypersomnia/sedation combined, oral hypoesthesia/dysgeusia, EPS, and incidence of clinically significant weight increase (defined as $\geq 7 \%$ increase from baseline to endpoint according to the US FDA 
guidance and product prescribing information) [9]. The Extrapyramidal Symptom Rating Scale (ESRS) was used to measure EPS [12]. The ESRS consisted of scores on EPSrelated items, leading to nine derived parameter scores. Ratings were made through a combination of clinical interview and motor examination. Further, events related to suicidality (e.g., suicidal ideation, suicidal behavior, and/or non-suicidal self-injurious behavior) were summarized based on responses to the C-SSRS at each visit [13].

Analysis of safety data also focused on mean changes from the OLE baseline in the following laboratory parameters: fasting glucose, fasting triglycerides, fasting cholesterol, prolactin, fasting insulin, and glycosylated hemoglobin $\left(\mathrm{HbA}_{1 \mathrm{c}}\right)$. Laboratory values were obtained at baseline and on days $28,126,182,266$, and 350. TEAEs potentially related to new-onset diabetes mellitus were analyzed by grouping for summary purposes hyperglycemia, new-onset diabetes mellitus, increased weight, increased appetite, increased blood glucose, central obesity, hyperlipidemia, and loss of consciousness as defined in the Medical Dictionary for Regulatory Activities (MedDRA) by the standardized MedDRA query (SMQ) 'hyperglycemia/new onset diabetes (broad)'. The criteria for metabolic syndrome (MBS) was defined by the International Diabetes Federation, which includes abdominal girth (measured throughout the trial, in centimeters) plus two other laboratory values (triglycerides, high-density lipoprotein cholesterol, blood pressure, or glucose), all of which had to be met at the same visit for a MBS diagnosis $[14,15]$. MBS was further categorized based on the study period (i.e., baseline and/or treatment periods).

\subsection{Effectiveness Outcomes}

The following select exploratory parameters were assessed during the study: change in YMRS total score from baseline(s) (pre-treatment and open-label); time to failure to maintain effect from open-label baseline to any time point (i.e., a $<50 \%$ reduction from baseline in YMRS total score for patients who achieved a $50 \%$ YMRS response during the acute trial and lost the effect during the OLE); total YMRS $50 \%$ responders and remitters (i.e., those who achieved $\geq 50 \%$ improvement in YMRS total score from each respective trial baseline to endpoint were considered responders, and remitters required a YMRS total score $\leq 12$ ); and change in CGI-BP severity scale overall score from OLE baseline. The YMRS is an 11-item clinicianrated scale to assess the severity and symptoms of manic episodes and can range from 0 (all symptoms absent) to 60 (all symptoms extreme) [16-18]. A decrease in YMRS total score is indicative of improved symptoms. The YMRS was administered at baseline and on days 28, 70, 126, 182, 266 , and 350 . The CGI-BP severity scale is a single-item clinician-rated scale with a possible score of $1-7$, where higher scores indicate greater severity [19]. The CGI-BP severity scale was administered on days $7,14,28,42,70$, $98,126,154,182,210,238,266,294,322$, and 350.

\subsection{Sample Size}

No formal sample size calculation was performed because this was a single-arm OLE trial and the primary objective was evaluation of safety and tolerability. The number of patients enrolled in this trial was dependent on the number of patients completing the double-blind acute trial and was expected to be $\geq 280$ patients (approximately $70 \%$ of patients enrolled in the acute trial).

\subsection{Statistical Analysis}

Analysis of safety data used the all-patients-as-treated (APaT) population, defined as all patients $\leq 17$ years old who received one or more dose of open-label trial medication. For analysis of safety parameters from the OLE baseline (or last day of the antecedent acute trial treatment), categorical variables were summarized as numbers and percentages. For continuous variables (such as changes from baseline), mean, median, minimum, and standard deviation (SD) were provided as summary measures. Additionally, within-group point estimates were provided. For the select TEAEs of interest and TEAEs that occurred in four or more patients overall, 2-sided $95 \%$ confidence intervals (CIs) were provided for the within-group percentage of patients with events using the 2-tailed exact binomial method proposed by Clopper-Pearson [20]. For continuous measures, 2-sided $95 \%$ CIs were provided for within-group differences using an analysis of covariance model with terms for pooled site, identified group, and baseline value (if applicable). Predefined limits of change were summarized using frequency tables for each visit and endpoint (if applicable) and shifts from baseline to postbaseline and endpoint (if applicable) by identified group.

Changes in body weight and body mass index (BMI) were also adjusted for growth (age and sex) and presented as mean Z-scores [21]. The Z-scores are assumed to follow a standard normal distribution and indicate how many SDs an observed value is away from the expected growth (i.e., weight, height, BMI), based on an individual's age and sex. Each Z-score corresponds to a percentile in the standardized growth curve. A Z-score of 0 corresponds to a 50th percentile (the median), whereas a negative Z-score corresponds to a percentile below the median and a positive $\mathrm{Z}$-score corresponds to a percentile above the median. The distance of a Z-score from 0 represents the distance of a percentile from the median. For the countries included in this trial (USA and Russia), age- and sex-adjusted growth 
was compared with expected growth seen in the general US population using the most recent growth charts at the time of database lock as provided by the National Center for Health Statistics (NCHS) [21]. A SAS program is available from the NCHS website that provides Z-scores and percentiles for weight, height, and BMI based on the US Centers for Disease Control and Prevention growth charts [21]. The Centers for Disease Control and Prevention reference database based on the US population may not be representative of sites outside the United States.

Efficacy analysis used the full analysis set population, defined as patients $\leq 17$ years old who received one or more dose of open-label trial medication and had one or more open-label postbaseline in-treatment YMRS total score. Efficacy results (YMRS total score, CGI-BP severity overall score, and total YMRS $50 \%$ responders) were evaluated using last observation carried forward (LOCF) and observed cases (OC) approaches. For LOCF analysis, the value of a particular assessment was the last available non-missing value prior to the particular assessment when the value was missing. In the OC approach, the assessment closest to the scheduled assessment day within the allowed window was used. If there were two assessments within the same absolute distance of the scheduled assessment, the one with the later date was chosen as OC. Kaplan-Meier analysis was used to estimate the time to failure to maintain response and was limited to those patients at endpoint in the acute trial who were considered total YMRS $50 \%$ responders. Days to failure to maintain response was defined as the number of days from first achieving responder status to the first subsequent time that the patient had less than a $50 \%$ reduction from open-label baseline in YMRS total score. A patient was considered a YMRS responder at a given visit if they experienced a $50 \%$ or more decrease from acute trial baseline in YMRS total score at that visit.

\section{Results}

\subsection{Patients}

A total of 350 patients completed the acute trial and 322 $(92.0 \%)$ were treated with open-label asenapine; 321 were included in the analyses (Fig. 1). As predefined analyses were limited to patients aged $\leq 17$ years at OLE entry, the one patient aged 18 years at entry was excluded from these analyses. Of the 321 included patients, 80 had been treated with placebo and 241 treated with asenapine in the antecedent acute phase trial. The mean age was $13.8 \pm 2.0$ years for the overall OLE population (Table 1). Other baseline demographic characteristics were similar between groups. The majority of patients in the total treatment group were White $(69.8 \%)$ and had a mean BMI of $24.6 \pm 6.3$. The most common comorbid Axis I disorder among the overall patient population was ADHD, which was diagnosed in $182(56.7 \%)$ patients, of whom 88 (48.4\%) were prescribed stimulants and $94(51.6 \%)$ were not. For the total treatment group, overall asenapine exposure ranged from 2 to 380 days, with a median duration of 182 days of treatment. Overall, 107 (33\%) patients were exposed to asenapine $2.5 \mathrm{mg}, 5 \mathrm{mg}$, or $10 \mathrm{mg}$ bid for at least 230 days. In the overall study population, 31 $(9.7 \%)$ patients received a modal dose of asenapine $2.5 \mathrm{mg}$ bid, $105(32.7 \%)$ patients received $5 \mathrm{mg}$ bid, and $170(53.0 \%)$ patients received $10 \mathrm{mg}$ bid. There were 15 (4.7\%) patients that received treatment for $<8$ days for which no modal dose was determined.

Concomitant medication use was reported by $69.8 \%$ of patients in the APaT population. The most commonly used medications were propionic acid derivatives (18.4\%; e.g., ibuprofen), sympathomimetics (16.5\%; e.g., methylphenidate), benzodiazepine derivatives (12.5\%; e.g., lorazepam), anilides (12.1\%; e.g., paracetamol), and mixed amphetamine salts $(10.3 \%)$.

\subsection{Safety}

No deaths occurred during the trial (Table 2). Overall, 267 $(83.2 \%)$ patients reported TEAEs in the total treatment group. Serious adverse events (SAEs) were reported in a total of $22(6.9 \%)$ patients. Most SAEs belonged to the psychiatric disorders class (i.e., suicidal ideation, aggression, bipolar disorder, depression, agitation). Overall, 13 $(4.0 \%)$ patients in the total treatment group discontinued treatment as a result of SAEs. The SAEs that led to discontinuation among these 13 patients included ADHD, aggression $(n=2)$, agitation, anxiety, bipolar disorder, depression, dystonia, intentional overdose, suicidal behavior, and suicidal ideation $(n=3)$. Additionally, 181 $(56.4 \%)$ patients discontinued from the total treatment group; the most common reasons were attributed to AEs $(48,15.0 \%)$, nonadherence to the study protocol (46, $14.3 \%)$, and consent withdrawal $(38,11.8 \%)$. The most common individual TEAEs leading to discontinuation in the overall population were somnolence $(1.9 \%)$, sedation $(1.2 \%)$, and fatigue $(1.2 \%)$.

Per the C-SSRS, 295 (91.9\%) of the 321 patients reported no positive responses (i.e., affirmative responses to questions on the C-SSRS) and one patient did not have C-SSRS data. Of the $25(7.8 \%)$ patients with positive responses on the C-SSRS, 23 (7.2\%) had some degree of suicidal ideation and/or behavior per the C-SSRS. The majority of the suicidal ideation was reported as passive or non-specific, or ideation without a plan or intent. A total of 8 of the $25(32.0 \%)$ patients with positive C-SSRS 
Fig. 1 Patient disposition. ${ }^{a}$ Discontinued and Completed consists of a mixture of patients who discontinued/completed the 26-week and 50-week versions of the protocol; ${ }^{b}$ the most common adverse events leading to treatment discontinuation were due to disease under study and occurred in 3 patients from the placebo/asenapine group and 10 patients from the asenapine/asenapine group (13 patients in total)

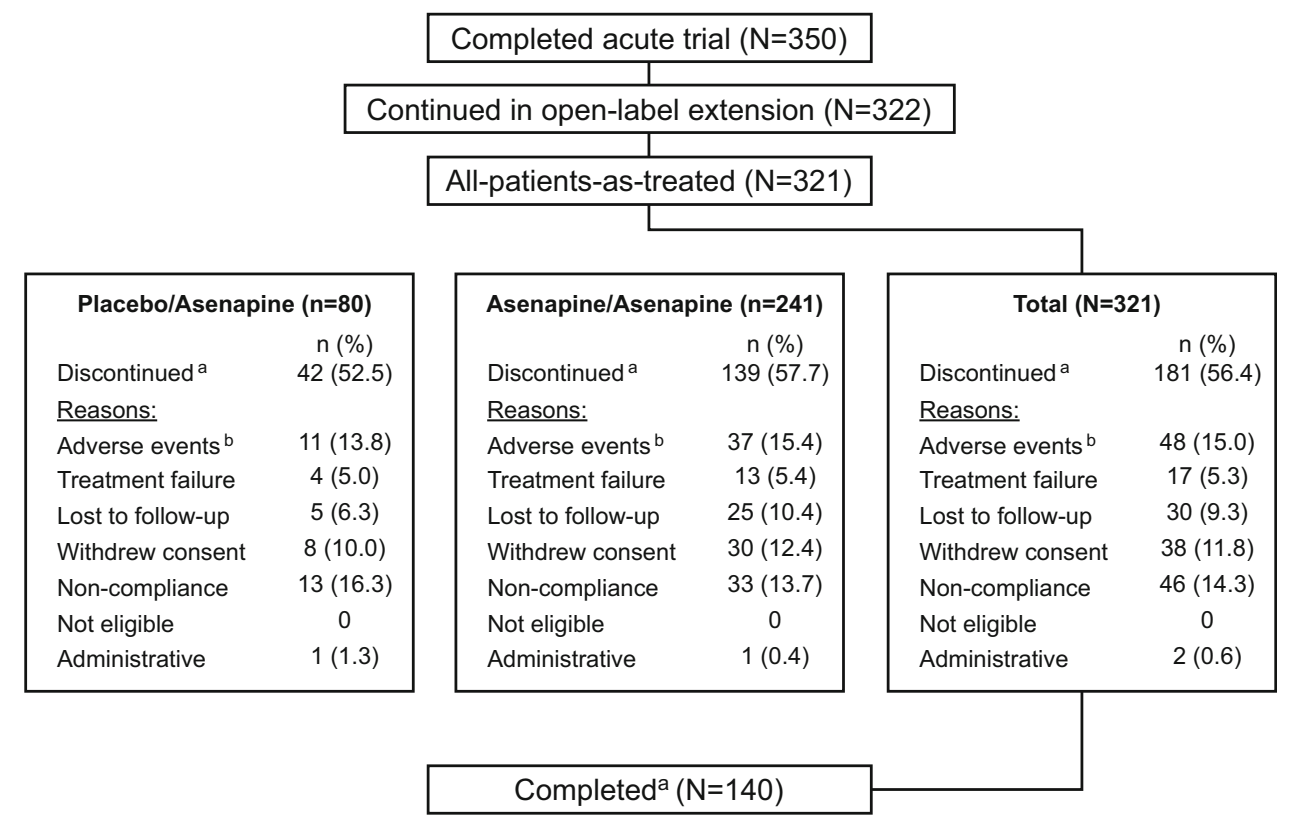

and adolescents are expected to gain weight and grow in height as part of normal development) as suggested by the observed increases in BMI. In the total treatment group, $109(34.8 \%)$ patients experienced clinically significant ( $\geq 7 \%$ ) weight gain (Table 2 ). TEAEs of weight gain were reported for $58(18.1 \%)$ patients. After correcting for age and sex, the mean weight and BMI scores at baseline and endpoint demonstrated an increase (Table 3). Weight category shifts were observed from the OLE baseline; more patients had upward shifts than downward shifts (see ESM, Online Resource 3). The mean change from baseline in weight percentile increased over time during the acute trial, and continued to increase initially during the OLE and appeared to stabilize temporally with continued treatment (see ESM, Online Resource 4). Results indicate that positive mean changes in BMI percentiles did not increase after week 18 (mean BMI percentile change of 3.9 at day 266) (see ESM, Online Resource 5). This is consistent with the corresponding weight percentile, where the maximum mean change in weight percentile occurred at week 18 (4.9 at day 266).

No clinically meaningful changes were noted in the laboratory parameters measured (Table 4). There were no patients with reported TEAEs with the specific preferred terms of hyperglycemia or new-onset diabetes. However, using the broad MedDRA definition, additional terms were included in the definition of new-onset diabetes mellitus (increased weight, increased appetite, increased blood glucose, central obesity, hyperlipidemia, and loss of consciousness). In the total treatment group, $73(22.7 \%)$ patients reported TEAEs within the broad scope of those pertaining to new-onset diabetes mellitus. Additionally, 
Table 1 Open-label extension baseline demographic and clinical characteristics of all patients aged 10-17 years with manic or mixed episodes associated with bipolar I disorder

\begin{tabular}{|c|c|c|c|}
\hline Parameter & $\begin{array}{l}\text { Placebo/ } \\
\text { asenapine } \\
N=80\end{array}$ & $\begin{array}{l}\text { Asenapine/ } \\
\text { asenapine } \\
N=241\end{array}$ & $\begin{array}{l}\text { Total } \\
N=321\end{array}$ \\
\hline Age, years, mean (SD) & $13.7(2.0)$ & $13.8(2.0)$ & $13.8(2.0)$ \\
\hline \multicolumn{4}{|l|}{ Sex, $n(\%)$} \\
\hline Male & $33(41.3)$ & $128(53.1)$ & $161(50.2)$ \\
\hline Female & $47(58.8)$ & $113(46.9)$ & $160(49.8)$ \\
\hline \multicolumn{4}{|l|}{ Race, $n(\%)$} \\
\hline White & $57(71.3)$ & $167(69.3)$ & $224(69.8)$ \\
\hline Black & $15(18.8)$ & $58(24.1)$ & $73(22.7)$ \\
\hline Asian, American Indian, Alaska Native, Native Hawaiian, or Other Pacific Islander & 0 & $5(2.1)$ & $5(1.6)$ \\
\hline Multiracial & $8(10.0)$ & $11(4.6)$ & $19(5.9)$ \\
\hline Weight, kg, mean (SD) & $66.8(23.7)$ & $63.8(20.3)$ & $64.5(21.2)$ \\
\hline BMI, kg/m², mean (SD) & $25.3(6.5)$ & $24.3(6.3)$ & $24.6(6.3)$ \\
\hline Abdominal girth, cm, mean (SD) & $82.4(16.1)$ & $80.4(15.7)$ & $80.9(15.8)$ \\
\hline \multicolumn{4}{|l|}{ Region, $n(\%)$} \\
\hline USA & $75(93.8)$ & $227(94.2)$ & $302(94.1)$ \\
\hline Non-USA & $5(6.3)$ & $14(5.8)$ & $19(5.9)$ \\
\hline \multicolumn{4}{|l|}{ Age of onset, years, at entry in the acute trial, $n(\%)$} \\
\hline$\leq 12$ & $25(31.3)$ & $68(28.2)$ & $93(29.0)$ \\
\hline$>12$ & $55(68.8)$ & $173(71.8)$ & $228(71.0)$ \\
\hline \multicolumn{4}{|l|}{ Comorbid ADHD, $n(\%)$} \\
\hline Yes, concomitant stimulant use, yes & $21(26.3)$ & $67(27.8)$ & $88(27.4)$ \\
\hline Yes, concomitant stimulant use, no & $24(30.0)$ & $70(29.0)$ & $94(29.3)$ \\
\hline No, concomitant stimulant use, yes & $1(1.3)$ & 0 & $1(0.3)$ \\
\hline No, concomitant stimulant use, no & $34(42.5)$ & $104(43.2)$ & $138(43.0)$ \\
\hline YMRS total score, mean $(\mathrm{SD})^{\mathrm{a}}$ & $20.2(8.7)$ & $15.3(8.7)$ & $16.5(9.0)$ \\
\hline CGI-BP overall score, mean $(\mathrm{SD})^{\mathrm{a}}$ & $3.7(0.9)$ & $3.1(1.0)$ & $3.2(1.0)$ \\
\hline
\end{tabular}

$A D H D$ attention-deficit hyperactivity disorder, $B M I$ body mass index, CGI-BP Clinical Global Impression scale for use in bipolar illness, $S D$ standard deviation, USA United States of America, YMRS Young Mania Rating Scale

a Placebo/asenapine $n=72$, asenapine/asenapine $n=227$, total $n=299$

there were two TEAEs of increased blood glucose and six cases of increased blood insulin; all of which were of mild or moderate intensity. In total, 18 patients met new-onset MBS criteria post-baseline during the extension study, whereas 10 patients who met MBS criteria at baseline did not meet MBS criteria at endpoint. A total of 12 patients met MBS at baseline and endpoint.

\subsection{Efficacy}

Change in YMRS total score from the acute trial baseline in this uncontrolled trial indicates that the improvement in mania, as measured by the mean change in YMRS total score from baseline, was maintained over the course of the extension trial for patients who were treated with asenapine in the acute trial (Fig. 2a). Mean change in YMRS total score from OLE baseline at week 26 was -6.9 points in the total treatment group and was of a greater magnitude in the placebo/asenapine group ( -13.0 points $)$ versus the asenapine/asenapine group ( -4.9 points). Week 50 results for total YMRS score were similar with a mean change of 9.2 points observed in the total treatment group, -15.2 in the placebo/asenapine group, and -6.5 in the asenapine/ asenapine group. Similar trends over time were observed with the LOCF approach.

Likewise, the change in CGI-BP severity overall score was similar among all treatment groups, indicating effectiveness was maintained over the course of the trial (Fig. 2b). The LOCF approach yielded consistent results.

Of the 141 patients who were total YMRS 50\% responders at the end of the acute trial and who were included in the OLE, 46 patients $(32.6 \%$ ) failed to 
Table 2 Summary of select TEAEs ${ }^{\mathrm{a}}$ in pediatric patients with bipolar I disorder

\begin{tabular}{|c|c|c|c|}
\hline & $\begin{array}{l}\text { Placebo/asenapine } \\
N=80 \\
n(\%)\end{array}$ & $\begin{array}{l}\text { Asenapine/asenapine } \\
N=241 \\
n(\%)\end{array}$ & $\begin{array}{l}\text { Total } \\
N=321 \\
n(\%)\end{array}$ \\
\hline Any TEAE ${ }^{\mathrm{b}}$ & $74(92.5)$ & $193(80.1)$ & $267(83.2)$ \\
\hline Death & 0 & 0 & 0 \\
\hline SAE & $5(6.3)$ & $17(7.1)$ & $22(6.9)$ \\
\hline Discontinuation caused by TEAE & $11(13.8)$ & $37(15.4)$ & $48(15.0)$ \\
\hline \multicolumn{4}{|l|}{$\geq 1$ predefined TEAE of interest during the study ${ }^{b}$} \\
\hline Somnolence, sedation, and hypersomnia combined & $48(60.0)$ & $88(36.5)$ & $136(42.4)$ \\
\hline Dizziness & $9(11.3)$ & $6(2.5)$ & $15(4.7)$ \\
\hline Insomnia & $2(2.5)$ & $6(2.5)$ & $8(2.5)$ \\
\hline Oral hypoesthesia and dysgeusia & $15(18.8)$ & $9(3.7)$ & $24(7.5)$ \\
\hline EPS $^{\mathrm{c}}$ & $6(7.5)$ & $14(5.8)$ & $20(6.2)$ \\
\hline Akathisia & $3(3.8)$ & $7(2.9)$ & $10(3.1)$ \\
\hline$\geq 7 \%$ weight increase from baseline to endpoint, $\mathrm{n} / \mathrm{n} 1(\%)$ & $28 / 75(37.3)$ & $81 / 238(34.0)$ & $109 / 313(34.8)$ \\
\hline \multicolumn{4}{|c|}{$\begin{array}{l}\text { EPS extrapyramidal symptom, } n 1 \text { number of patients available for assessment, } O L E \text { open-label extension, } S A E \text { serious adverse event, } T E A E \\
\text { treatment-emergent adverse event }\end{array}$} \\
\hline
\end{tabular}

Table 3 Z-scores ${ }^{a}$ for weight and BMI asenapine-treated pediatric patients with bipolar I disorder

\begin{tabular}{llll}
\hline Z-score for age and sex, mean (SD) & $\begin{array}{l}\text { Placebo/asenapine } \\
N=80\end{array}$ & $\begin{array}{l}\text { Asenapine/asenapine } \\
N=241\end{array}$ & $\begin{array}{l}\text { Total } \\
N=321\end{array}$ \\
\hline Weight, kg & & & $0.93(1.19)$ \\
Baseline & $1.11(1.16)$ & $0.91(1.18)$ & $0.98(1.17)$ \\
Endpoint & $1.21(1.14)$ & $0.08(0.36)$ & $0.08(0.35)$ \\
Change & $0.10(0.32)$ & & $0.94(1.12)$ \\
BMI, kg/m ${ }^{2}$ & & $0.88(1.15)$ & $1.00(1.15)$ \\
Baseline & $1.15(0.98)$ & $0.93(1.19)$ & $0.06(0.45)$ \\
Endpoint & $1.25(0.98)$ & $0.05(0.49)$ & \\
Change $^{\mathrm{b}}$ & $0.10(0.31)$ & &
\end{tabular}

$B M I$ body mass index, $S D$ standard deviation

${ }^{a} \mathrm{Z}$-score indicates how many SDs a value is away from the expected value for a person of the same sex and age. A Z-score of 0 corresponds to the 50th percentile (i.e., the median), a negative Z-score corresponds to a percentile below the median, and a positive Z-score corresponds to a percentile above the median. Percentiles are corrected for age and sex

b Change from baseline to endpoint was calculated using the open-label baseline

maintain effect in the OLE. At day 182 (week 26), the Kaplan-Meier estimate of the cumulative percentage of failures to maintain effect was $31.1 \%$ in the total treatment group.

After 26 weeks of treatment in the OLE, a total of 118 of $149(79.2 \%)$ patients were total YMRS $50 \%$ responders using the acute trial baseline; YMRS remission (YMRS total score $\leq 12$ ) was reached by 102 of 149 $(68.5 \%)$ patients at the end of treatment in the OLE. Of note, 26-week data were used owing to the limited number of patients with data values after week 26 .

\section{Discussion}

Flexibly dosed asenapine $(2.5 \mathrm{mg}, 5 \mathrm{mg}$, and $10 \mathrm{mg}$ bid) was generally well tolerated in pediatric patients with an acute manic or mixed episode associated with BP-1 treated 
Table 4 Mean (SD) change from baseline ${ }^{a}$ in laboratory parameters of interest in asenapine-treated pediatric patients with bipolar I disorder

\begin{tabular}{llll}
\hline & $\begin{array}{l}\text { Placebo/asenapine } \\
N=80\end{array}$ & $\begin{array}{l}\text { Asenapine/asenapine } \\
N=241\end{array}$ & $N=321$ \\
\hline Fasting cholesterol, $\mathrm{mg} / \mathrm{dL}$ & $2.67(22.93)$ & $-3.40(22.31)$ & $-1.89(22.54)$ \\
Fasting triglycerides, $\mathrm{mg} / \mathrm{dL}$ & $0.62(50.97)$ & $-0.97(58.76)$ & $-0.53(56.81)$ \\
Fasting glucose, $\mathrm{mg} / \mathrm{dL}$ & $0.90(13.33)$ & $1.26(13.69)$ & $1.26(13.51)$ \\
$\mathrm{HbA}_{1 \mathrm{c}}(\geq 7 \%), n$ & 0 & 0 & 0 \\
Fasting insulin, $\mu \mathrm{U} / \mathrm{mL}$ & $4.51(19.72)$ & $-6.35(55.72)$ & $-3.58(49.27)$ \\
Prolactin, $\mathrm{ng} / \mathrm{mL}$ & $2.31(12.30)$ & $-0.39(14.07)$ & $0.27(13.69)$ \\
\hline
\end{tabular}

$H b A_{l c}$ glycosylated hemoglobin, $S D$ standard deviation

a Mean changes and shifts were calculated using the open-label extension baseline

for up to 50 weeks. Somnolence $(26.8 \%)$, weight gain $(18.1 \%)$, sedation $(15.9 \%)$, and headache $(12.8 \%)$ were the most commonly reported individual TEAEs and somnolence, sedation, and fatigue were the most common TEAEs leading to discontinuation in the $\operatorname{OLE}(1.9,1.2$, and $1.2 \%$, respectively). These TEAEs, combined with dizziness and oral hypoesthesia, occurred more frequently in asenapine-naïve patients and may, therefore, be TEAEs to consider monitoring with initiation of asenapine in this population. Additionally, SAEs that led to discontinuation included ADHD, aggression, agitation, anxiety, dystonia, intentional overdose, suicidal behavior, and suicidal ideation. These SAEs are as expected for a long-term, openlabel study in this patient population, making it difficult to interpret if, or how, they are related to the study medication. That said, during the acute trial the incidence of these SAEs was higher in those randomized to receive asenapine compared with placebo [11].

A total of $34.8 \%$ of patients experienced clinically significant weight increase (defined as $\geq 7 \%$ increase in body weight at endpoint based on the current US FDA guidance and product label). Weight gain is common with other atypical antipsychotics in children and adolescents [22]. However, it should be noted that children and adolescents are expected to experience a degree of weight gain that is attributable to normal growth, especially considering the duration of this long-term trial. In the present study, the overall mean (SD) weight gain at endpoint was 3.5 (5.8) $\mathrm{kg}$. Weight gain based on weight percentile (adjusted for growth, as based on age and sex) continued to increase after treatment initiation in this extension trial, which is consistent with alterations observed in fasting metabolic parameters (i.e., total cholesterol, high-density lipoprotein, low-density lipoproteins, triglycerides, glucose, and insulin) in a minority of patients at study endpoint. Results of patients meeting criteria for MBS suggest that asenapine may be temporally associated with development of MBS in some patients. Therefore, metabolic monitoring should be performed in pediatric patients initiating therapy with asenapine and, in addition, healthy lifestyle instruction should accompany treatment.

An average improvement in the YMRS total score and CGI-BP severity score was seen over time in this uncontrolled trial. In addition, patients who transitioned to asenapine in the OLE after receiving placebo during the acute phase essentially achieved the same level of response as the asenapine/asenapine group by the end of the extension study. Of note, almost one third of patients who met the criterion for response (50\% improvement in YMRS) at the end of the acute trial failed to sustain this level of improvement at all visits during the OLE. This may be a reflection of the strict maintenance criterion since patients were assessed as having failed to maintain response even if the drop below the $50 \%$ improvement threshold was temporary and response was regained at subsequent visits. Additionally, since fluctuation of symptoms in bipolar disorder may be more common in pediatric patients than in adult patients [5], patients who failed to maintain response at every assessment may have experienced symptom fluctuation that is characteristic of the pediatric course of the disorder. However, after 26 weeks of open-label asenapine treatment, $79 \%$ of patients were YMRS $50 \%$ responders and $68 \%$ of patients achieved YMRS remission (YMRS total score $\leq 12$ ), suggesting that a large proportion of patients achieved clinically meaningful symptom improvement.

\subsection{Limitations}

Although this study had a relatively large sample size, it was an open-label study and efficacy and safety results should be interpreted with caution. In addition, over half of the patients in this study discontinued prematurely which may limit generalizability of these results; however, the completion rate $(44.6 \%)$ in this study was similar to that seen in other long-term studies of antipsychotics in adolescent populations [23-25] and may be related to the length of the trial and the ages of the patients. Furthermore, 
Fig. 2 Efficacy during the open-label extension (OC, full analysis set). a Least squares mean change from acute phase baseline in YMRS total score to end of treatment by visit; b Mean (SD) change in CGI-BP overall score from acute phase baseline to end of treatment by visit. The CGI-BP overall score ranged from 1 (normal, not at all ill) to 7 (among the most extremely ill patients). Decreases from baseline within a treatment group were indicative of an improvement. The YMRS total score could range from 0 (all symptoms absent) to 60 (all symptoms extreme). Decreases from baseline within a treatment group are indicative of an improvement in symptoms. Full analysis set is all patients $\leq 17$ years of age who received $\geq 1$ dose of trial medication and had both baseline and $\geq 1$ postbaseline in-treatment YMRS total scores in the acute and extension trial. CGI-BP Clinical Global Impression scale for use in bipolar illness, $O C$ observed case, $S D$ standard deviation, YMRS Young Mania Rating Scale

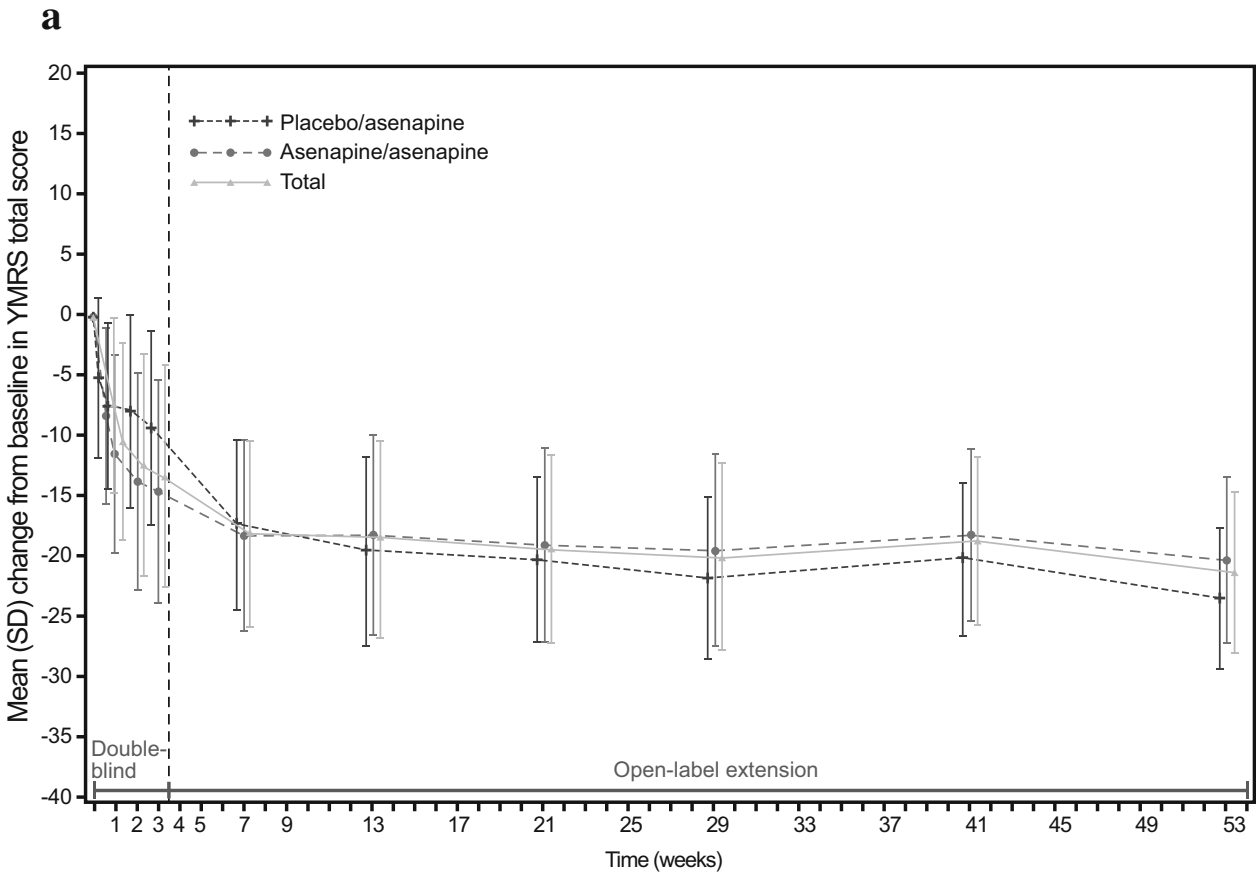

b

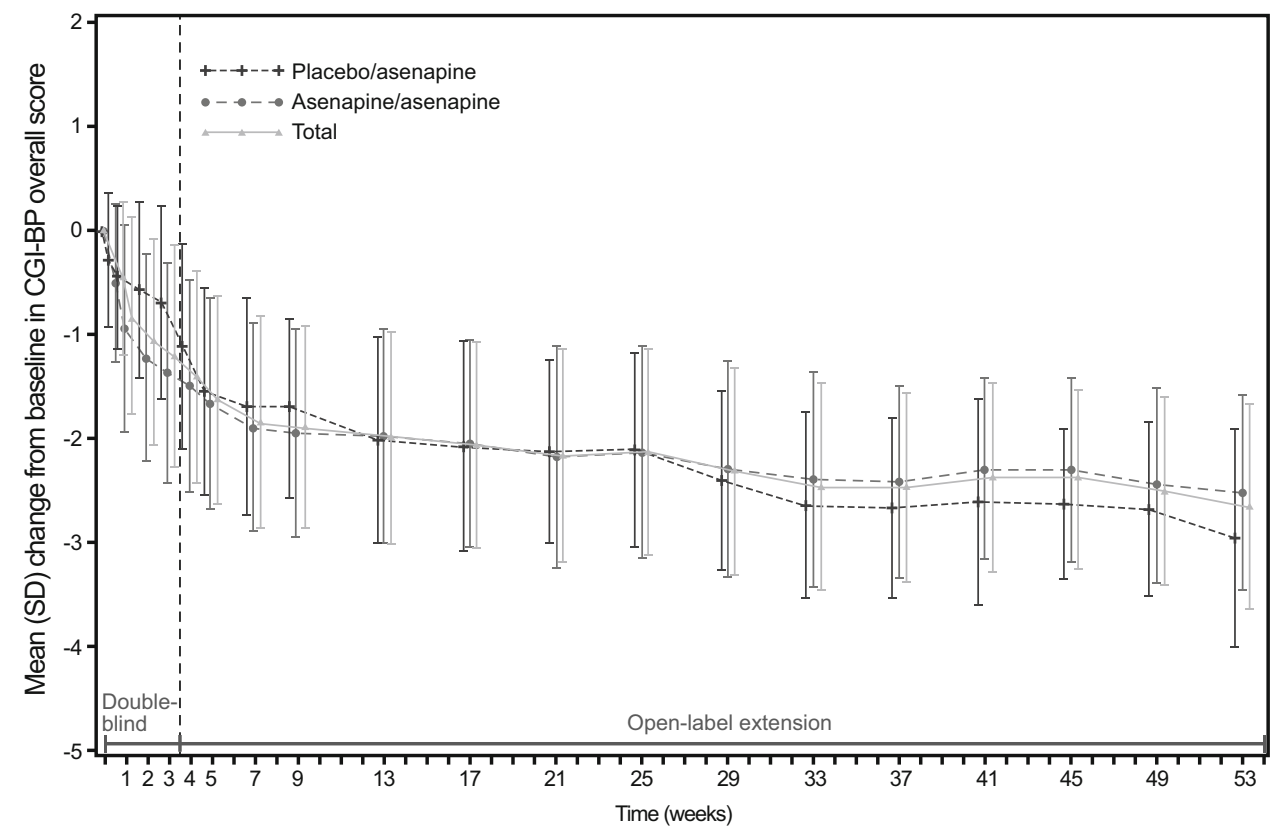

results may not be generalizable to the entire child and adolescent population with BP-1 due to the inclusion and exclusion criteria used in this study. While not a limitation of the study per se, accurate diagnosis of BP-1 in pediatric patients may be challenging; symptoms of bipolar illness can be seen in other populations without bipolarity [26]. In the DSM, fifth edition, increased energy has been added into the 'A criterion' for the diagnosis of the manic features specifier which is more inclusive than the DSM-IV-TR diagnosis of manic episode and requires at least three opposite pole symptoms [27]. It is therefore unclear how this may affect results of clinical trials that previously enrolled patients on the basis of DSM-IV-TR criteria.

\section{Conclusions}

Long-term therapy with asenapine was generally well tolerated in pediatric patients aged 10-17 years with BP-1, although $15.0 \%$ of patients discontinued due to TEAEs 
and $34.8 \%$ experienced clinically significant $(\geq 7 \%)$ weight gain with long-term asenapine therapy. Further studies are warranted in children and adolescents with BP1 to improve our understanding of weight gain and other metabolic abnormalities associated with long-term asenapine treatment, and their relation to the normal changes that occur in this patient population during growth. The combination of somnolence, sedation, and hypersomnia was the most commonly reported TEAE of interest. Somnolence and sedation were also among the most common TEAEs leading to discontinuation and may be treatmentlimiting in a minority of patients.

Acknowledgments The authors thank Sabine Braat and Janelle Koppenhaver, formerly with Merck (Oss, The Netherlands), for their important contributions to the study. Tonya Goodman, Arbor Communications, Ann Arbor, MI, USA provided medical writing services in the preparation of this manuscript. All authors had full access to the data after study completion and unblinding and are responsible for the work described in this paper. All authors were involved in at least one of the following: conception, design, acquisition, analysis, and statistical analysis, interpretation of data, and drafting the manuscript and/or revising the manuscript for important intellectual content. All authors provided final approval of the version to be published.

\section{Compliance with Ethical Standards}

Funding The study was designed by Merck \& Co., Inc., which had direct oversight or participation in every stage of the study. Funding for medical writing services was provided by Allergan, Inc.

Disclosure of potential conflict of interest Findling: receives or has received research support, acted as a consultant and/or served on a speaker's bureau for Alcobra, American Academy of Child \& Adolescent Psychiatry, American Physician Institute, American Psychiatric Press, AstraZeneca, Bracket, Bristol-Myers Squibb, CogCubed, Cognition Group, Coronado Biosciences, Dana Foundation, Elsevier, Forest, GlaxoSmithKline, Guilford Press, Johns Hopkins University Press, Johnson and Johnson, Jubilant Clinsys, KemPharm, Lilly, Lundbeck, Merck, NIH, Neurim, Novartis, Noven, Otsuka, Oxford University Press, Pfizer, Physicians Postgraduate Press, Purdue, Rhodes Pharmaceuticals, Roche, Sage, Shire, Sunovion, Supernus Pharmaceuticals, Transcept Pharmaceuticals, Tris Pharma, Validus, and WebMD.

Landbloom, Mackle, Snow-Adami: employees of Merck \& Co., may own stock or have stock options.

$W u$, Durgam: employees of Allergan, may own stock or have stock options.

Chang: receives or has received research support, or has acted as a consultant for GlaxoSmithKline, Actavis, Lilly, Sunovion, Janssen, and Merck.

Research involving human participants All procedures performed in studies involving human participants were in accordance with the ethical standards of the institutional and/or national research committee and with the 1964 Helsinki declaration and its later amendments or comparable ethical standards.

Informed consent Informed consent was obtained from all individual participants included in the study.
Open Access This article is distributed under the terms of the Creative Commons Attribution-NonCommercial 4.0 International License (http://creativecommons.org/licenses/by-nc/4.0/), which permits any noncommercial use, distribution, and reproduction in any medium, provided you give appropriate credit to the original author(s) and the source, provide a link to the Creative Commons license, and indicate if changes were made.

\section{References}

1. Merikangas KR, Cui L, Kattan G, Carlson GA, Youngstrom EA, Angst J. Mania with and without depression in a community samples of US adolescents. Arch Gen Psychiatry. 2012;69: 943-51.

2. Van Meter AR, Moreira AL, Youngstrom EA. Meta-analysis of epidemiologic studies of pediatric bipolar disorder. J Clin Psychiatry. 2011;72:1250-6.

3. Freeman AJ, Youngstrom EA, Michalak E, Siegel R, Meyers OI, Findling RL. Quality of life in pediatric bipolar disorder. Pediatrics. 2009;123:e446-52.

4. Perlis RH, Miyahara S, Marangell LB, Wisniewski SR, Ostacher M, DelBello MP, et al. Long-term implications of early onset bipolar disorder: data from the first 1000 participants in the systematic treatment enhancement program for bipolar disorder (STEP-BD). Biol Psychiatry. 2004;55:875-81.

5. Birmaher B, Axelson D, Strober M, Gill MK, Valeri S, Chiappetta L, et al. Clinical course of children and adolescents with bipolar spectrum disorders. Arch Gen Psychiatry. 2006;63: 175-83.

6. Perlis RH, Dennehy EB, Miklowitz DJ, Delbello MP, Ostacher M, Calabrese JR, et al. Retrospective age at onset of bipolar disorder and outcome during two-year follow-up: results from the STEP-BD study. Bipolar Disord. 2009;11:391-400.

7. Post RM, Leverich GS, Kupka RW, Keck PE Jr, McElroy SL, Altshuler LL, et al. Early-onset bipolar disorder and treatment delay are risk factors for poor outcome in adulthood. J Clin Psychiatry. 2010;71:864-72.

8. Bourgeois FT, Murthy S, Pinto C, Olson KL, Ioannidis JP, Mandl KD. Pediatric versus adult drug trials for conditions with high pediatric disease burden. Pediatrics. 2012;130:285-92.

9. SAPHRIS [package insert] St. Louis: Actavis Inc.; 2015.

10. Caccia S. Safety and pharmacokinetics of atypical antipsychotics in children and adolescents. Pediatr Drugs. 2013;15:217-33.

11. Findling RL, Landbloom RL, Szegedi A, Koppenhaver J, Braat S, Zhu Q, et al. Asenapine for the acute treatment of pediatric manic or mixed episode of bipolar I disorder. J Am Acad Child Adolesc Psychiatry. 2015;54:1032-41.

12. Chouinard G, Margolese HC. Manual for the Extrapyramidal Symptom Rating Scale (ESRS). Schizophr Res. 2005;76:247-65.

13. Posner K, Brown GK, Stanley B, Brent DA, Yershova KV, Oquendo MA, et al. The Columbia-Suicide Severity Rating scale: initial validity and internal consistency findings from three multisite studies with adolescents and adults. Am J Psychiatry. 2011;168:1266-77.

14. Alberti KG, Zimmet P, Shaw J. The metabolic syndrome-a new worldwide definition. Lancet. 2005;366:1059-62.

15. Alberti KG, Zimmet P, Shaw J. The metabolic syndrome in children and adolescents. Lancet. 2007;369:2059-61.

16. Young R, Biggs JT, Ziegler VE, Meyer DA. A rating scale for mania: reliability, validity and sensitivity. $\mathrm{Br} \mathrm{J}$ Psychiatry. 1978;133:429-35. 
17. Fristad MA, Weller EB, Weller RA. The Mania Rating Scale: can it be used in children? A preliminary report. J Am Acad Child Adolesc Psychiatry. 1992;31:252-7.

18. Youngstrom EA, Danielson CK, Findling RL, Gracious BL, Calabrese JR. Factor structure for the Young Mania Rating Scale for use with youths ages 5 to 17 years. J Clin Child Adolesc Psychol. 2002;31:567-72.

19. Spearing MK, Post RM, Leverich GS, Brandt D, Nolen W. Modification of the Clinical Global Impressions (CGI) scale for use in bipolar illness (BP): the CGI-BP. Psychiatry Res. 1997;73: 159-71.

20. Clopper CJ, Pearson ES. The use of confidence or fiducial limits illustrated in the case of the binomial. Biometrika. 1934;26: 404-12.

21. Centers for Disease Control and Prevention: a SAS Program for the 2000 CDC Growth Charts (ages 0 to $<20 \mathrm{y}$ ), 2014. http://www.cdc.gov/nccdphp/dnpao/growthcharts/resources/sas. htm. Accessed 23 July 2015.

22. De Hert M, Dobbelaere M, Sheridan EM, Cohen D, Correll CU. Metabolic and endocrine adverse effects of second-generation antipsychotics in children and adolescents: a systematic review of randomized, placebo controlled trials and guidelines for clinical practice. Eur Psychiatry. 2011;26:144-58.
23. Findling RL, Cavuş I, Pappadopulos E, Vanderburg DG, Schwartz JH, Gundapaneni BK, DelBello MP. Efficacy, longterm safety, and tolerability of ziprasidone in children and adolescents with bipolar disorder. J Child Adolesc Psychopharmacol. 2013;23:545-57.

24. Findling RL, Cavuş I, Pappadopulos E, Vanderburg DG, Schwartz JH, Gundapaneni BK, DelBello MP. Ziprasidone in adolescents with schizophrenia: results from a placebo-controlled efficacy and long-term open-extension study. J Child Adolesc Psychopharmacol. 2013;23:531-44.

25. Findling RL, Correll CU, Nyilas M, Forbes RA, McQuade RD, Jin N, Ivanova S, Mankoski R, Carson WH, Carlson GA. Aripiprazole for the treatment of pediatric bipolar I disorder: a 30-week, randomized, placebo-controlled study. Bipolar Disord. 2013;15:138-49.

26. Findling RL, Youngstrom EA, Fristad MA, Birmaher B, Kowatch RA, Arnold LE, et al. Characteristics of children with elevated symptoms of mania: the Longitudinal Assessment of Mania Symptoms (LAMS) study. J Clin Psychiatry. 2010;71:1664-72.

27. American Psychiatric Association. Diagnostic and Statistical Manual of Mental Disorders. 5th ed. Arlington: American Psychiatric Association; 2013. 